\title{
Lower Bounds for Resonance Widths in Potential and Obstacle Scattering
}

\author{
Claudio Fernandez ${ }^{1 \star}$ and Richard Lavine ${ }^{2 \star \star}$ \\ 1 Facultad de Matematicas, Pontificia Universidad Catolica de Chile, Casilla 6177, Santiago, Chile \\ 2 Department of Mathematics, University of Rochester, Rochester, NY 14627, USA
}

\begin{abstract}
Explicit lower bounds are given for the size of the imaginary parts of resonances for Schrödinger operators with non-trapping or trapping potentials, and for the Dirichlet Laplacian in the exterior of a star-shaped obstacle, both acting in three dimensions.
\end{abstract}

\section{Introduction}

Resonances for perturbations of the Laplace operator $\Delta$ on $\mathbb{R}^{n}$ are of interest in the theory of scattering for the Schrödinger equation

$$
\frac{\partial \psi(x, t)}{\partial t}=-i(-\Delta+V(x)) \psi(x, t) \quad x \in \mathbb{R}^{n}, t \in \mathbb{R}
$$

and the wave equation outside an obstacle $\Omega$

$$
\frac{\partial^{2} u(x, t)}{\partial t^{2}}=\Delta u(x, t) \quad x \in \mathbb{R}^{n} \backslash \Omega, t \in \mathbb{R} .
$$

They are associated with abnormally long, but temporary trapping of quantum mechanical particles for (1.1), or waves for (1.2). Mathematically, a self adjoint perturbation $H$ of $-\Delta$ is said to have a resonance $k=\kappa-i \eta \in \mathbb{C}$ if its resolvent $(H-z)^{-1}$ has an analytic continuation in $z$ with a pole at $k^{2}$. This gives a solution $\psi$ of the eigenvalue equation $H \psi=k^{2} \psi$ which also satisfies an outgoing radiation condition at $\infty$. (This condition is incompatible with square integrability, so $k^{2}$ is not an eigenvalue.)

Such a solution $\psi$ gives a solution $\psi(x, t)=\exp \left(-i k^{2} t\right) \psi(x)$ of $(1.1)$ and a solution $u(x, t)=\exp (-i k t) \psi(x)$ of (1.2). The approximate lifetimes of these are respectively $(2 \kappa \eta)^{-1}$ and $\eta^{-1}$. Suppose the perturbation is supported in $\mathscr{B}_{R}=\{|x| \leqq R\}$. The time spent by an unperturbed particle or wave in $\mathscr{B}_{R}$ is

\footnotetext{
$\star$ Work partially supported by DiUC/FONDECYT (Chile)

$\star \star$ Work partially supported by U.S. National Science Foundation grant DMS 8705610
} 
roughly $2 R /$ velocity, which is $2 R / 2 \kappa$ for (1.1) and $2 R$ for (1.2). Thus for both equations, the lifetime is "long" if $2 \eta R \ll 1$. If the perturbation is of a non-trapping type, we would expect to find no resonances obeying this inequality.

Star-shaped obstacles do not trap light rays, and potentials satisfying $E-V-r \partial V / \partial r>0$ do not trap classical particles of energy $E$. In this paper we show that in dimension 3 such perturbations, if supported in $\mathscr{B}_{R}$, do not produce resonances with $\eta R$ very small and $\kappa R$ not too small. For $\kappa$ large the condition becomes approximately $4.72 \eta R<1$.

If the non-trapping conditions fail, resonances with $\eta R \ll 1$ are expected. For the potential case we give lower bounds which are exponentially small in a certain quantity which roughly measures the size of the trapping barrier.

Resonance free regions of the complex plane are known to exist for the Laplacian in the exterior of an obstacle. An explicit lower bound $\eta>$ const. was found in $[\mathrm{M}]$ for a class of obstacles including star-shaped ones, and lower bounds were shown to exist for non-trapping obstacles in $[\mathrm{M}-\mathrm{R}-\mathrm{S}]$. The sphere of radius $R$ has a resonance at $k=-i / R$. The strip $\eta R \geqq 1$ has been shown to be resonance-free in $[R]$, a reference we learned about after completing this work. Our explicit lower bound for $\eta$ is weaker than the result of [R], but the method is different, and should apply in even dimensions. It is shown in $[B-L-R]$ that for convex obstacles the lower bound grows as $\kappa^{1 / 3}$.

For the Schrödinger equation, less seems to be known. In [B-C-D, S, D-H] lower bounds on $\eta$ are given for non-trapping $V$ in the semiclassical limit, i.e. a given lower bound for $\eta$ is found to hold as Planck's constant $h$ approaches zero.

Our bounds cannot recover such results, since they are $O(h)$ at best. However they yield explicit results for a given potential. Explicit bounds were found in $[\mathrm{H}]$ for general (possibly trapping) potentials in one dimension. In [LO] an explicit resonance-free strip below the real axis is given for a class of non-trapping potentials in three dimensions. In this paper we give explicit energy dependent lower bounds on $\eta$, separately for the non-trapping and trapping cases, both for potentials of compact support in $\mathbb{R}^{3}$. Our energy dependent non-trapping bounds apply to any reasonable potential, since there will be no trapping at high energy.

Our proof is elementary, and similar in spirit to those of [H] and [LO]. The main ideas are demonstrated by the following results in one dimension. The basic estimate for the non-trapping case uses the method of [LA-1] first developed for resolvent estimates (and thus estimates on lifetimes). The modification for trapping potentials was used in [LA-2, LA-3]. If $V$ has compact support a resonance in one dimension for $-d^{2} / d x^{2}+V$ on $[0, \infty)$ is just a $k$ such that there is a solution of $-\varphi^{\prime \prime}+V \varphi=k^{2} \varphi$ with $\varphi(r)=c e^{-i k r}$ for $r$ outside the support of $V$.

Theorem 1.1. Suppose that $V \in \mathscr{C}^{1}([0, \infty))$, supp $V \subset[0, R]$, and the operator $H=-d^{2} / d x^{2}+V$ on $[0, \infty)$ with Dirichlet boundary condition at 0 has a resonance at $k=\kappa-i \eta$ with $\kappa, \eta>0$. Then

$$
2 \eta R \geqq \frac{\inf \left\{\kappa^{2}-V-r V^{\prime}-\frac{2}{(2 R)^{2}}: x \in[0, R]\right\}}{\sup \left\{\kappa^{2}+\frac{1}{(2 R)^{2}}+V: x \in[0, R]\right\}} .
$$


Proof. Suppose that $-\varphi^{\prime \prime}+V \varphi=k^{2} \varphi$, with $\varphi(0)=0$, and $\varphi(x)=e^{i k x}$ for $x \geqq R$. Then, using integration by parts we find

$$
\begin{aligned}
0= & \operatorname{Re} \int_{0}^{R} x \bar{\varphi}^{\prime}\left[-\varphi^{\prime \prime}+\left(V-k^{2}\right) \varphi\right] d x \\
= & \frac{1}{2} \int_{0}^{R}\left|\varphi^{\prime}\right|^{2} d x-\frac{R}{2}\left|\varphi^{\prime}(R)\right|^{2}+\frac{1}{2} \int_{0}^{R} \operatorname{Re}\left(k^{2}-V-x V^{\prime}\right)|\varphi|^{2} d x \\
& -\frac{R \operatorname{Re} k^{2}}{2}-|\varphi(R)|^{2}+\operatorname{Im}(\kappa-i \eta)^{2} \int_{0}^{R} x \operatorname{Im} \bar{\varphi}^{\prime} \varphi d x .
\end{aligned}
$$

Now

$$
\int_{0}^{R} x \operatorname{Im} \bar{\varphi}^{\prime} \varphi d x=\int_{0}^{R} x \frac{\bar{\varphi}^{\prime} \varphi-\bar{\varphi} \varphi^{\prime}}{2 i} d x=\int_{0}^{R} \frac{x^{2}-R^{2}}{2} 2 \kappa \eta|\varphi|^{2} d x
$$

so the last term in (1.4) is non-negative. Since $\varphi(x)=c e^{i k x}$ for $x \geqq R$ we have $\varphi^{\prime}(R)-i k \varphi(R)=0$. Therefore

$$
\begin{aligned}
& \int_{0}^{R}\left|\varphi^{\prime}\right|^{2}+\left(\operatorname{Re} k^{2}-V-x V^{\prime}\right)|\varphi|^{2} d x \\
& \quad \leqslant R\left[\left|\varphi^{\prime}(R)\right|^{2}+\left(\kappa^{2}+\eta^{2}\right)|\varphi(R)|^{2}\right) \\
& \quad=R\left|\varphi^{\prime}(R)-i k \varphi(R)\right|^{2}+2 \kappa R \operatorname{Im} \varphi^{\prime}(R) \overline{\varphi(R)}+2 \eta R \operatorname{Re} \varphi^{\prime}(R) \overline{\varphi(R)} \\
& =2 \kappa R \operatorname{Im} \varphi^{\prime}(R) \overline{\varphi(R)}+2 \eta R \operatorname{Re} \varphi^{\prime}(R) \overline{\varphi(R)} \\
& =R \int_{0}^{R} \frac{d}{d x}\left(2 \kappa \operatorname{Im} \varphi^{\prime} \bar{\varphi}+2 \eta \operatorname{Re} \varphi^{\prime} \bar{\varphi}\right) d x \\
& =R \int_{0}^{R}\left[4 \kappa^{2} \eta|\varphi|^{2}+2 \eta\left|\varphi^{\prime}\right|^{2}+2 \eta\left(V-\kappa^{2}+\eta^{2}\right)|\varphi|^{2}\right] d x \\
& \quad=2 \eta R \int_{0}^{R}\left[\left(\kappa^{2}+V+\eta^{2}\right)|\varphi|^{2}+\left|\varphi^{\prime}\right|^{2}\right] d x .
\end{aligned}
$$

Suppose that (1.3) is violated. then $2 \eta R<1$ (since $V$ and $V^{\prime}$ vanish at $R$ ) and therefore $\eta^{2} \leqq 1 /(2 R)^{2}$, so $(1.5)$ implies $(1.3)$.

Remark. If $\kappa^{2}>V(x)$ for $x \in[0, R]$, then by solving an ordinary differential equation we can find $g(x)$ such that $g^{\prime}(x) \geqq 1$ and $g^{\prime}(x)\left(\kappa^{2}-V\right)-g(x) V^{\prime} \geqq \kappa^{2}$. Replacing $x$ by $g(x)$ in the proof of Theorem 1.1 yields a more general lower bound for $\eta$ :

$$
2 \eta g(R) \geqq \frac{\kappa^{2}-1 /(2 g(R))^{2}}{\sup \left\{\kappa^{2}+V(x)+1 /(2 g(R))^{2}: x \in[0, R]\right\}} .
$$

(In general $g(R)$ will be larger, and thus the bound smaller, the more $V$ oscillates.)

Notice that (1.3) is trivial unless $\kappa^{2}-V-r V^{\prime} \geqq 0$. This condition is sufficient to rule out classical trapped orbits at energy $\kappa^{2}$. If this condition is violated, there may be trapped orbits, and resonances near the real axis. It is possible to bound 
the imaginary part of such a resonance from below by the following trick. Suppose that for some $R_{1}>R$,

$$
u\left(R_{1}\right)=1, \quad u^{\prime}\left(R_{1}\right)=0, \quad u^{\prime}(r)<0, \quad\left(r<R_{1}\right) .
$$

If $-\varphi^{\prime \prime}+V \varphi=k^{2} \varphi$, then $\varphi / u$ satisfies

$$
-\left(u^{2}\left(\frac{\varphi}{u}\right)^{\prime}\right)^{\prime}+u^{2}\left(V(x)-\frac{u^{\prime \prime}}{u}-k^{2}\right) \frac{\varphi}{u}=0 .
$$

So, writing $q=u^{\prime \prime} / u$, we have, as in Theorem 1.1

$$
\begin{aligned}
\operatorname{Re} \int_{0}^{R_{1}} \frac{x}{u^{2}}\left(\frac{\bar{\varphi}}{u}\right)^{\prime}\left[-\left(u^{2}\left(\frac{\varphi}{u}\right)^{\prime}\right)^{\prime}+u^{2}\left(V-q-k^{2}\right) \frac{\varphi}{u}\right] d x \\
\quad-\frac{1}{2} \int_{0}^{R_{1}}\left\{\left|\left(\frac{\varphi}{u}\right)^{\prime}\right|^{2}\left(1-4 \frac{u^{\prime}}{u}\right)+\left(\operatorname{Re} k^{2}-V+q-x(V-q)^{\prime}\right)\left|\frac{\varphi}{u}\right|^{2}\right\} d x \\
\quad+\frac{1}{2}\left[\left[\left.\varphi^{\prime}(R)\right|^{2}+\left(\kappa^{2}-\eta^{2}\right)|\varphi(R)|^{2}\right]\right. \\
=\int_{0}^{R_{1}} \frac{x}{u^{2}} \operatorname{Im} k^{2} u^{2} \operatorname{Im}\left(\frac{\bar{\varphi}}{u}\right)^{\prime} \frac{\varphi}{u} d x \\
=\operatorname{Im} k^{2} \int_{0}^{R_{1}} \int_{x}^{R_{1}} \frac{y}{u^{2}(y)} d y\left[u^{2} \operatorname{Im}\left(\frac{\bar{\varphi}^{\prime}}{u} \frac{\varphi}{u}\right)\right]^{\prime} d x \\
=\left(\operatorname{Im} k^{2}\right)^{2} \int_{0}^{R_{1}} \int_{x}^{R_{1}} \frac{y}{u^{2}(y)} d y u^{2}\left|\frac{\varphi}{u}\right|^{2} d x \geqq 0 .
\end{aligned}
$$

Now

$$
\begin{aligned}
\int_{0}^{R_{1}}\left|\left(\frac{\varphi}{u}\right)^{\prime}\right|^{2} d x & =\int_{0}^{R_{1}} \frac{1}{u^{2}}\left[\left|\varphi^{\prime}\right|^{2}-2 \frac{u^{\prime}}{u} \operatorname{Re} \varphi^{\prime} \bar{\varphi}+\left|\frac{u^{\prime}}{u}\right|^{2}|\varphi|^{2}\right] d x \\
& \geqq \frac{1}{\|u\|_{\infty}^{2}} \int_{0}^{R_{1}}\left(\left|\varphi^{\prime}\right|^{2}+q \|\left.\varphi\right|^{2}\right) d x,
\end{aligned}
$$

so we obtain, as in Theorem 1.1

$$
\begin{aligned}
& \int_{0}^{R_{1}}\left\{\left|\varphi^{\prime}\right|^{2}+\left[\left(\kappa^{2}-\eta^{2}-(x(V-q))^{\prime}\right]|\varphi|^{2}\right\} d x\right. \\
& \quad \leqq\|u\|_{\infty}^{2} 2 \eta R_{1} \int_{0}^{R_{1}}\left\{\left|\varphi^{\prime}\right|^{2}+\left(\kappa^{2}+\eta^{2}+V\right)|\varphi|^{2}\right\} d x
\end{aligned}
$$

which is impossible if $\eta$ is too small. A bound $\|u\|_{\infty}$ is obtained in Lemma 2.7. (With the simpler argument of $[\mathrm{H}]$ we must estimate $\varphi$. Here we must estimate $u$, a function which may be chosen to improve the estimate on $\eta$. We do not pursue this here.)

In the rest of the paper, we will extend these ideas to 3 dimensions. The integration by parts argument which we give in Sect. 2 is quite similar, but to be 
effective a certain change of variables from $\psi$ to $r \psi$ is necessary. Most of the difficulties, however, arise from the more complicated behavior of outgoing solutions of $\left(\Delta+k^{2}\right) \psi=0$. We cannot expect that $r \psi=\exp i k r$, but $(\partial / \partial r-i k)(r \psi)$ is small. The proof treats separately the cases of small and large angular momentum. In Sect. 3, it is shown that for components with small angular momentum $l$ this quantity is small relative to $r^{-1} l|r \psi|$, while in Sect. 4 we show that the components with large angular momentum are small in size. A better argument for this part of the proof would be welcome. In Sect. 5 the resulting lower bounds are derived.

\section{Interior Estimates}

In this section we consider a solution $\psi$ of

$$
\begin{aligned}
\nabla \cdot \gamma(x) \nabla \psi(x)+\lambda(x) \psi(x) & =0, \quad x \in B_{\rho} \backslash \Omega \subset \mathbb{R}^{n}, \\
\psi(x) & =0, \quad x \in \partial \Omega,
\end{aligned}
$$

where $\Omega$ is a star-shaped obstacle, for real $\gamma$ and complex $\lambda$ in $\mathscr{C}_{0}^{1}\left(\mathscr{B}_{\rho} \backslash \Omega\right)$. We shall essentially estimate the $\mathscr{H}_{1}$-norm of $\psi$ over $\mathscr{B}_{\rho} \backslash \Omega$ and the $L^{2}$ norm of its normal derivative on $\partial \Omega$, in terms of its Cauchy data on $\{|x|=\rho\}$. Here we allow arbitrary dimension $n$, but for simplicity we eventually restrict to $n=3$.

Proposition 2.1. Let $\mathscr{R}$ be a bounded domain in $\mathbb{R}^{n}$ with $\mathscr{C}^{1}$ boundary, $\gamma \in \mathscr{C}^{1}(\mathscr{R})$ and $G \in \mathscr{C}^{2}(\mathscr{R})$ both real and $\lambda \in \mathscr{C}^{1}(\mathscr{R})$ and $\varphi \in \mathscr{C}^{2}(\mathscr{R})$ complex. Then

$$
\begin{aligned}
\operatorname{Re} \int & \nabla G \cdot \nabla \bar{\varphi}(-\nabla \cdot \gamma \nabla \varphi-\lambda \varphi) d x \\
= & \int_{\mathscr{R}}\left\{\gamma \operatorname{Hess} G(\nabla \varphi, \nabla \varphi)-\frac{1}{2} \nabla \cdot(\gamma \nabla G)|\nabla \varphi|^{2}+\frac{1}{2} \operatorname{Re}[\nabla \cdot(\lambda \nabla G)]|\varphi|^{2}\right\} d x \\
& \quad-\int_{\partial \mathscr{R}}\left\{\gamma(\nabla G \cdot \nabla \bar{\varphi})(\nabla \varphi \cdot v)-\frac{\gamma}{2} \nabla G \cdot v|\nabla \varphi|^{2}+\frac{1}{2} \nabla G \cdot v \operatorname{Re} \lambda|\varphi|^{2}\right\} d S \\
& -\int_{\mathscr{R}} \operatorname{Im} \lambda \operatorname{Im}(\nabla G \cdot \nabla \varphi \bar{\varphi}) d x,
\end{aligned}
$$

where

$$
\text { Hess } G(v, v)=\sum_{i, j=1}^{n} \frac{\partial^{2} G}{\partial x_{i} \partial x_{j}} \bar{v}_{i} v_{j}, \quad v=\left(v_{1}, \ldots, v_{n}\right) \in \mathbb{C}^{n} \text {. }
$$

Proof. Integration by parts yields

$$
\begin{aligned}
\int_{\mathscr{R}} \nabla G \cdot \nabla \bar{\varphi}(-\nabla \cdot \gamma \nabla \varphi) d x \\
=\int_{\mathscr{R}}\left\{\gamma \operatorname{Hess} G(\nabla \varphi, \nabla \varphi)+\sum_{i=1}^{n} \gamma \nabla G \cdot\left(\nabla \frac{\partial \bar{\varphi}}{\partial x_{i}}\right) \frac{\partial \varphi}{\partial x_{i}}\right\} d x \\
\quad-\int_{\partial \mathscr{R}} \gamma(\nabla G \cdot \nabla \bar{\varphi})(\nabla \varphi \cdot v) d S,
\end{aligned}
$$


and for arbitrary real $f$ and $\theta$

$$
\begin{aligned}
\int_{\mathscr{R}} \nabla G \cdot \nabla \bar{\theta} f \theta d x= & -\int_{\mathscr{R}} \nabla \cdot(f \nabla G)|\theta|^{2} d x-\int_{\mathscr{R}} \nabla G \cdot \nabla \theta f \bar{\theta} d x \\
& +\int_{\partial \mathscr{R}} \nabla G \cdot v f|\theta|^{2} d S,
\end{aligned}
$$

which implies

$$
\operatorname{Re} \int_{\mathscr{R}} \nabla G \cdot \nabla \bar{\theta} f \theta d x=-\frac{1}{2} \int_{\mathscr{R}} \nabla \cdot(f \nabla G)|\theta|^{2} d x+\frac{1}{2} \int_{\partial \mathscr{R}} \nabla G \cdot v f|\theta|^{2} d S .
$$

Applying this with $f=\gamma, \theta=\partial \varphi / \partial x_{i}$ to the second term on the right side of (2.3) and also to the remaining term on the left side of (2.2) with $\theta=\varphi$ and $f=\operatorname{Re} \lambda$ gives (2.2).

In our applications, $\operatorname{Im} \lambda$ will always be a constant multiple of $\gamma$.

Proposition 2.2. If $0<g \in \mathscr{C}^{1}(\mathbb{R})$ is real and $\varphi$ satisfies $(2.1)$, and $\operatorname{Im} \lambda / \gamma$ is constant, then

$$
\begin{aligned}
\int_{\mathscr{B}_{\rho} \backslash \Omega} & \left\{\gamma\left(g^{\prime}(r)-\frac{g(r)}{r}\right)\left|\frac{\partial \varphi}{\partial r}\right|^{2}+\frac{1}{2}\left(g \frac{\gamma}{r}(3-n)-\frac{\partial \gamma}{\partial r} g-\gamma g^{\prime}\right)|\nabla \varphi|^{2}\right. \\
& \left.+\frac{1}{2} \operatorname{Re}\left[\lambda g^{\prime}(r)+\frac{n-1}{r} \lambda g(r)+\frac{\partial \lambda}{\partial r} g(r)\right]|\varphi|^{2}\right\} d x \\
& +\frac{1}{2} \int_{\partial \Omega} \gamma g(r) \hat{x} \cdot v|\nabla \varphi|^{2} d S \\
\leqq & \frac{1}{2} \int_{|x|=\rho}\left\{\gamma g\left[\left|\frac{\partial \varphi}{\partial r}\right|^{2}-\left(|\nabla \varphi|^{2}-\left|\frac{\partial \varphi}{\partial r}\right|^{2}\right)\right]+g \operatorname{Re} \lambda|\varphi|^{2}\right\} d S,
\end{aligned}
$$

where $v$ denotes the outword normal to $\partial \Omega$ here.

Proof. Take $G(x)=-\int_{|x|}^{\rho} g(s) d s$ so that

$$
\nabla G(x)=g(r) \hat{x}, \quad \Delta G=g^{\prime}(r)+\frac{n-1}{r} g(r)
$$

and

$$
\text { Hess } G(v, v)=\left(g^{\prime}-\frac{g}{r}\right)|\hat{x} \cdot v|^{2}+\frac{g}{r}|v|^{2} \text {. }
$$

Then (2.4) follows from (2.2) and the observations that on $\partial \Omega,|\nabla \varphi|=|\partial \varphi / \partial v|$, and

$$
\begin{aligned}
\int_{\mathscr{B} \rho} \backslash \Omega & \operatorname{Im} \lambda \operatorname{Im}(\nabla G \cdot \nabla \varphi \bar{\varphi}) d x \\
= & -\int_{\mathscr{B} \supset \backslash \Omega}\left[G \nabla\left(\frac{\operatorname{Im} \lambda}{\gamma}\right) \cdot \gamma \operatorname{Im} \nabla \varphi \bar{\varphi}+G \frac{\operatorname{Im} \lambda}{\gamma} \nabla \cdot \gamma \frac{\nabla \varphi \bar{\varphi}-\varphi \nabla \bar{\varphi}}{2 i}\right] d x
\end{aligned}
$$




$$
=\int_{\mathscr{B}_{\rho} \backslash \Omega} G \frac{(\operatorname{Im} \lambda)^{2}}{\gamma}|\varphi|^{2} d x \leqq 0,
$$

because $G(r) \leqq 0$ for $r \leqq \rho$ and $G(r)=0$ for $r=\rho$.

If $\Omega$ is star-shaped, then $\hat{x} \cdot v \geqq 0$ on $\partial \Omega$ so the boundary integral on the left-hand side of (2.4) is non-negative. But to get a useful estimate we need the integral over $\mathscr{B}_{\rho} \backslash \Omega$ positive. The choice $g=r$ requires

$$
\begin{aligned}
(2-n) \gamma-r \frac{\partial \gamma}{\partial r} & \geqq 0, \\
\operatorname{Re}\left(n \lambda+r \frac{\partial \lambda}{\partial r}\right) & \geqq 0 .
\end{aligned}
$$

For the important case $\gamma=1,(2.5)$ fails if $n>2$.

However, we can make use of the fact, well known in certain circles, that one can trade off between $\gamma$ and $\lambda$. In fact, for $u>0$ and smooth,

$$
\begin{aligned}
\nabla \cdot(\gamma \nabla \varphi) & =\nabla \cdot\left(\frac{1}{u} u^{2} \gamma \frac{1}{u} \nabla \varphi\right) \\
& =\frac{1}{u}\left(\nabla-\frac{\nabla u}{u}\right) \cdot\left[u^{2} \gamma\left(\nabla+\frac{\nabla u}{u}\right) \frac{\varphi}{u}\right] \\
& =\frac{1}{u}\left\{\nabla \cdot\left(u^{2} \gamma \nabla \frac{\varphi}{u}\right)+u \nabla \cdot \gamma \nabla u \frac{\varphi}{u}\right\}
\end{aligned}
$$

so that $\varphi / u$ satisfies an equation of the form (2.1). For example, if $\psi$ satisfies (2.1) with $\gamma=1$, and $u=r^{-(n-1) / 2}$, then

$$
\frac{\nabla \cdot \gamma \nabla u}{u}=\frac{-(n-1)(n-3)}{4 r^{2}}
$$

and $\varphi=r^{(n-1) / 2} \psi$ satisfies

$$
0=\left\{\nabla \cdot \frac{1}{r^{n-1}} \nabla+\frac{\lambda}{r^{n-1}}-\frac{(n-1)(n-3)}{4 r^{n+1}}\right\} \varphi .
$$

Now the quantities in (2.5) and (2.6) become $r^{-(n-1)}$ and

$$
\operatorname{Re}\left(\lambda+r \partial \lambda / \partial r+(n-1)(n-3) / 4 r^{2}\right) r^{-(n-1)} \text {. }
$$

Thus we obtain

Theorem 2.3. Let $\Omega$ be a domain in $\mathbb{R}^{n}$ (possibly empty) with $\mathscr{C}^{1}$-boundary. Suppose that $\psi$ satisfies the Schrödinger equation

$$
\begin{gathered}
-\Delta \psi+V \psi=k^{2} \psi, \quad x \in \mathscr{B}_{\rho} \backslash \Omega, \\
\psi(x)=0, \quad x \in \partial \Omega,
\end{gathered}
$$


where $V \in \mathscr{C}^{1}\left(\mathbb{R}^{n}\right)$, supp $V \subset \mathscr{B}_{\rho}$, and $k=\kappa-$ in. Then $\varphi=r^{(n-1) / 2} \psi$ satisfies

$$
\begin{aligned}
& \int_{B_{\rho} \backslash \Omega}\left\{|\nabla \varphi|^{2}+\left[\kappa^{2}-\eta^{2}-V-r \frac{\partial V}{\partial r}+\frac{(n-1)(n-3)}{4 r^{2}}\right]|\varphi|^{2}\right\} \frac{d x}{r^{n-1}}+\int_{\partial \Omega} x \cdot v|\nabla \varphi|^{2} \frac{d S}{r^{n-1}} \\
& \leqq \frac{1}{\rho^{n-2}} \int_{r=\rho}\left\{\left|\frac{\partial \varphi}{\partial r}\right|^{2}-\left(|\nabla \varphi|^{2}-\left|\frac{\partial \varphi}{\partial r}\right|^{2}\right)+\left[\kappa^{2}-\eta^{2}-\frac{(n-1)(n-3)}{4 r^{2}}\right]|\varphi|^{2}\right\} d S .
\end{aligned}
$$

In the next two sections it is shown that in dimension 3 , if $\varphi=r \psi$, where $\psi$ is a resonance eigenfunction, then $\partial \varphi / \partial r-i k \varphi$ cannot be too large at suitable values of $\rho$. The following result shows that this quantity cannot be too small if $\psi$ satisfies (2.8). (A similar result holds for $n \geqq 3$.)

Lemma 2.4. Suppose that $n=3$, and $\varphi$ and $\psi$ are as in Theorem 2.3. Then

$$
\begin{aligned}
\frac{1}{\rho_{|x|=\rho}} \int_{\partial r}\left|\frac{\partial \varphi}{\partial r}-i k \varphi\right|^{2} d S= & \frac{1}{\rho_{|x|=\rho}}\left\{\left|\frac{\partial \varphi}{\partial r}\right|^{2}+|k|^{2}|\varphi|^{2}\right\} d S \\
& -2 \pi \rho \int_{\mathscr{B}_{\rho} \backslash \Omega}\left\{|\nabla \varphi|^{2}+\left(\kappa^{2}+V+\eta^{2}\right)|\varphi|^{2}\right\} \frac{d x}{r^{2}} .
\end{aligned}
$$

Proof. We have

$$
\left|\frac{\partial \varphi}{\partial r}-i k \varphi\right|^{2}=\left|\frac{\partial \varphi}{\partial r}\right|^{2}+|k|^{2}|\varphi|^{2}-2 \kappa \operatorname{Im} \frac{\partial \varphi}{\partial r} \bar{\varphi}-2 \eta \operatorname{Re} \frac{\partial \varphi}{\partial r} \bar{\varphi}
$$

and

$$
\begin{aligned}
\frac{1}{\rho^{2}} \int_{r=\rho} \frac{\partial \varphi}{\partial r} \bar{\varphi} d S & =\int_{r=\rho} \frac{1}{r^{2}} \nabla \varphi \cdot v \bar{\varphi} d S \\
& =\int_{\mathscr{B}_{\rho} \backslash \Omega} \nabla \cdot\left(\frac{1}{r^{2}} \nabla \varphi \bar{\varphi}\right) d S \\
& =\int_{\mathscr{P}_{\rho} \backslash \Omega}\left\{\left(V-k^{2}\right)|\varphi|^{2}+|\nabla \varphi|^{2}\right\} \frac{d x}{r^{2}} .
\end{aligned}
$$

Taking real and imaginary parts, we obtain

$$
\begin{aligned}
& \frac{1}{\rho} \int_{|x|=\rho} 2 \kappa \operatorname{Im} \frac{\partial \varphi}{\partial r} \bar{\varphi} d S=4 \kappa^{2} \eta \rho \int_{\mathscr{B} \rho \backslash \Omega}|\varphi|^{2} d x, \\
& \frac{1}{\rho_{|x|=\rho}} 2 \eta \operatorname{Re} \frac{\partial \varphi}{\partial r} \bar{\varphi} d S=2 \eta \rho \int_{\mathscr{B} \supset \backslash \Omega}\left\{\left(V-\kappa^{2}+\eta^{2}\right)|\varphi|^{2}+|\nabla \varphi|^{2}\right\} \frac{d x}{r^{2}},
\end{aligned}
$$

from which the conclusion follows.

Combining this with Theorem 2.3 gives the following. 
Corollary 2.5. In dimension 3 , with $\varphi$ and $\psi$ as in Theorem 2.3, we have

$$
\begin{aligned}
& \int_{\mathscr{B} \supset \backslash \Omega}\left\{(1-2 \eta \rho)|\nabla \varphi|^{2}+\left[\kappa^{2}(1-2 \eta \rho)-(1+2 \eta \rho)\left(V+\eta^{2}\right)-r \frac{\partial V}{\partial r}\right]|\varphi|^{2}\right\} \frac{d x}{r^{2}} \\
& +\int_{\partial \Omega} x \cdot v|\nabla \varphi|^{2} \frac{d S}{r^{2}} \leqq \frac{1}{\rho_{r}} \int_{r=\rho}\left\{\left|\frac{\partial \varphi}{\partial r}-i k \varphi\right|^{2}-\left(|\nabla \varphi|^{2}-\left|\frac{\partial \varphi}{\partial r}\right|^{2}\right)\right\} d S .
\end{aligned}
$$

This result gives the desired estimate for small $\eta$ if $\kappa^{2}-V-r \partial V / \partial r>0$. This condition is sufficient to rule out classical bound orbits at energy $\kappa^{2}$. When it fails, resonances near the real axis may exist. In [LA-2, 3] an upper bound was obtained for sojourn times near the scatterer. Here we use the same technique to get an inequality like (2.12), whose left-hand side is positive if $\eta$ is (very) small. The idea is that if $H$ is a Schrödinger operator whose potential $V$ supports a classical bound orbit at energy $E$, it may be equivalent at energy $E$, via (2.7) to another differential operator whose symbol represents a classical Hamiltonian that does not trap.

In (2.7), take $\gamma=r^{-(n-1)}$ and $u=u(r)$. Then

$$
\nabla \cdot \frac{1}{r^{n-1}} \nabla \varphi=\frac{1}{u}\left(\nabla \cdot \frac{u^{2}}{r^{n-1}} \nabla \frac{\varphi}{u}+\frac{u^{\prime \prime}(r) u(r)}{r^{n-1}} \frac{\varphi}{u}\right),
$$

so if $\psi$ satisfies (2.8), we have, writing $q=u^{\prime \prime} / u$,

$$
\nabla \cdot\left(\frac{u^{2}}{r^{n-1}} \nabla \frac{\varphi}{u}\right)+\frac{u^{2}}{r^{n-1}}\left(k^{2}-V+q(r)\right) \frac{\varphi}{u}=0 .
$$

Thus the inequalities (2.5) and (2.6) required for an estimate become

$$
\begin{gathered}
\frac{u^{2}}{r^{n-1}}\left(1-2 r \frac{u^{\prime}}{u}\right) \geqq 0, \\
\frac{u^{2}}{r^{n-1}}\left(\kappa^{2}-\eta^{2}-V-r \frac{\partial V}{\partial r}+q(r)+r q^{\prime}(r)\right) \geqq 0 .
\end{gathered}
$$

In fact it is possible to choose $u$ so that $u^{\prime} / u \leqq 0$ and the new potential $V-q$ does not trap at energy $\kappa^{2}$.

Theorem 2.6. Suppose that $V \in \mathscr{C}^{1}\left(\mathbb{R}^{3}\right)$ and $\operatorname{supp} V \subset \mathscr{B}_{\boldsymbol{R}}$ and that $h \in \mathscr{C}([0, \infty))$ satisfies

$$
V(x)+r \frac{\partial V}{\partial r}(x) \leqq h(|x|)
$$

with $h$ decreasing and $h(r)=0$ for $r>R$. Set

$$
R_{1}=\max \left\{R, \frac{2}{\kappa^{2}} \int_{0}^{R} h(s) d s\right\}
$$


and let

$$
q(r)=\left\{\begin{array}{ll}
\frac{1}{r} \int_{0}^{r} h(s) d s-\frac{1}{R_{1}} \int_{0}^{R} h(s) d s & r<R_{1} \\
0 & r \geqq R_{1}
\end{array},\right.
$$

if $k=\kappa-$ in and $\psi$ satisfies (2.8) then $\varphi=r \psi$ satisfies, for $\rho>R_{1}$

$$
\begin{aligned}
& \int_{\mathscr{B}_{\rho}}\left\{|\nabla \varphi|^{2}+\left(\frac{\kappa^{2}}{2}-\eta^{2}\right)|\varphi|^{2}\right\} \frac{d x}{r^{2}} \\
& \leqq B \int_{|x|=\rho}\left\{\left|\frac{\partial \varphi}{\partial r}\right|^{2}-\left(|\nabla \varphi|^{2}-\left|\frac{\partial \varphi}{\partial r}\right|^{2}\right)+\left(\kappa^{2}-\eta^{2}\right)|\varphi|^{2}\right\} \frac{d S}{\rho},
\end{aligned}
$$

and if $2 \eta \rho B \leqq 1$

$$
\int_{\mathscr{B}_{\rho}}\left(\frac{\kappa^{2}}{2}-\eta^{2}-2 \eta \rho B\right)|\varphi|^{2} \frac{d x}{r^{2}} \leqq B \int_{|x|=\rho}\left\{\left|\frac{\partial \varphi}{\partial r}-i k \varphi\right|^{2}-\left(|\nabla \varphi|^{2}-\left|\frac{\partial \varphi}{\partial r}\right|^{2}\right)\right\} \frac{d S}{\rho},
$$

where

$$
B=\cosh ^{2} \int_{0}^{R_{1}} q(s)^{1 / 2} d s
$$

First we require a result on ordinary differential equations.

Lemma 2.7. Suppose that, for $0<r<r_{0}$,

$$
q(r) \geqq 0, \quad q^{\prime}(r) \leqq 0, \quad \text { and } \quad q\left(r_{0}\right)=0 .
$$

Let $u(r)$ satisfy

$$
\begin{aligned}
& u^{\prime \prime}(r)=q(r) u(r), \quad 0<r<r_{0}, \\
& u\left(r_{0}\right)=1, \quad u^{\prime}\left(r_{0}\right)=0 .
\end{aligned}
$$

Then for $0 \leqq r \leqq r_{0}, u^{\prime}(r) \leqq 0$, and

$$
1 \leqq u(r) \leqq \cosh \int_{r}^{r_{0}} q(s)^{1 / 2} d s .
$$

Proof. The function $v=u^{\prime} / u$ satisfies

$$
v^{\prime}(r)=q(r)-v(r)^{2}, \quad 0<r<r_{0} ; \quad v\left(r_{0}\right)=0,
$$

while the functions

$$
v_{-}(r)=0 \text { and } v_{+}(r)=q(r)^{1 / 2} \tanh \left(\int_{r_{0}}^{r}(q(s))^{1 / 2} d s\right)
$$

satisfy the same initial condition at $r_{0}$, and

$$
\begin{aligned}
& v_{-}^{\prime}(r)=0 \leqq q-v_{-}^{2}, \\
& v_{+}^{\prime}(r)=\frac{1}{2} q^{\prime}(r) / q(r)^{1 / 2} \tanh \left(\int_{r_{0}}^{r}(q(s))^{1 / 2} d s\right)+q(r) \operatorname{sech}^{2}\left(\int_{r_{0}}^{r}(q(s))^{1 / 2} d s\right) \geqq q(r)-v_{+}^{2} .
\end{aligned}
$$


So by comparison, $v_{-}(r) \geqq v(r) \geqq v_{+}(r)$, i.e.

$$
0 \geqq \frac{u^{\prime}(r)}{u(r)} \geqq q(r)^{1 / 2} \tanh \int_{r_{0}}^{r} q(s)^{1 / 2} d s .
$$

Then (2.17) follows by integration and exponentiation.

Proof of Theorem 2.6. Define $u(r)$ by

$$
\begin{aligned}
& u^{\prime \prime}(r)=q(r) u(r), \quad r<\rho, \\
& u(\rho)=1, \quad u^{\prime}(\rho)=0 .
\end{aligned}
$$

Note that $q$ is decreasing and non-negative, and vanishes at $R_{1}$, so Lemma 2.7 applies to $u$. We have seen that $\varphi(x) / u(r)$ satisfies (2.13).

We may apply Proposition 2.2 to $\varphi / u$, (with $\Omega=\varnothing$ ) since

$$
\frac{\operatorname{Im} \lambda}{\gamma}=\frac{-2 \kappa \eta u^{2} / r^{n-1}}{u^{2} / r^{n-1}}=-2 \kappa \eta .
$$

Taking $g(r)=r / u(r)^{2}$, we get

$$
\begin{gathered}
\int_{B_{\rho}}\left\{\left|\nabla \frac{\varphi}{u}\right|^{2}-4 \frac{u^{\prime}}{u}\left|\frac{\partial \varphi / u}{\partial r}\right|^{2}+\left[\kappa^{2}-\eta^{2}-\frac{\partial}{\partial r}(r V-r q(r))\right]\left|\frac{\varphi}{u}\right|^{2}\right\} \frac{d x}{r^{2}} \\
\leqq \frac{1}{\rho_{r}} \int_{r=\rho}\left\{\left|\frac{\partial \varphi}{\partial r}\right|^{2}-\left(|\nabla \varphi|^{2}-\left|\frac{\partial \varphi}{\partial r}\right|^{2}\right)+\left(\kappa^{2}-\eta^{2}\right)|\varphi|^{2}\right\} d S .
\end{gathered}
$$

By definition of $q$ and $R_{1}$, we have, for $r<R_{1}$,

$$
\begin{aligned}
\frac{\partial}{\partial r}(r q(r)) & =h(r)-\frac{1}{R_{1}} \int_{0}^{R} h(s) d s \\
& \geqq V+r \frac{\partial V}{\partial r}-\frac{\kappa^{2}}{2} .
\end{aligned}
$$

Therefore the coefficient of $|\varphi / u|^{2}$ on the left side of (2.18) exceeds $\kappa^{2} / 2-\eta^{2}$. Also

$$
\begin{aligned}
\int_{\mathscr{B}_{\rho}}\left|\nabla \frac{\varphi}{u}\right|^{2} \frac{d x}{r^{2}} & =\int_{\mathscr{B}_{\rho}} \frac{1}{u^{2}}\left|\nabla \varphi-\frac{u^{\prime}}{u}-\frac{x}{r} \varphi\right|^{2} \frac{d x}{r^{2}} \\
& =\int_{\mathscr{B}_{\rho}} \frac{1}{u^{2}}\left\{|\nabla \varphi|^{2}-2 \frac{u^{\prime}}{u} \operatorname{Re} \frac{x}{r} \cdot \nabla \varphi \bar{\varphi}+\left|\frac{u^{\prime}}{u} \varphi\right|^{2}\right\} \frac{d x}{r^{2}} \\
& \geqq \frac{1}{\|u\|_{\infty}^{2}}\left\{\int_{\mathscr{B}_{\rho}}\left[|\nabla \varphi|^{2}+\left|\frac{u^{\prime}}{u} \varphi\right|^{2}+r^{2} \nabla \cdot\left(\frac{u^{\prime}}{u} \frac{x}{r^{3}}\right)|\varphi|^{2}\right] \frac{d x}{r^{2}}-\int_{|x|=\rho} \frac{u^{\prime}}{u}|\varphi|^{2} \frac{d S}{r^{2}}\right\} \\
& =\frac{1}{\|u\|_{\infty}^{2}} \int_{\mathscr{B}_{\rho}}\left[|\nabla \varphi|^{2}+q(r)|\varphi|^{2}\right] \frac{d x}{r^{2}} .
\end{aligned}
$$

We have used $u^{\prime}\left(R_{1}\right)=0$ in the last step. Using this again, and the fact that, by 
Lemma 2.7, $u^{\prime}(r) / u(r) \leqq 0$ for $r<R_{1}$, we obtain from (2.18) and (2.17),

$$
\begin{aligned}
& \int_{\mathscr{B}_{\rho}}\left[|\nabla \varphi|^{2}+\left(\frac{\kappa^{2}}{2}-\eta^{2}+q(r)\right)|\varphi|^{2}\right] \frac{d x}{r^{2}} \\
& \leqq B \int_{r=\rho}\left\{\left|\frac{\partial \varphi}{\partial r}\right|^{2}-\left(|\nabla \varphi|^{2}-\left|\frac{\partial \varphi}{\gamma r}\right|^{2}\right)+\left(\kappa^{2}-\eta^{2}\right)|\varphi|^{2}\right\} \frac{d S}{\rho},
\end{aligned}
$$

which implies (2.15). Also (2.16) follows from (2.15) and (2.11).

\section{Estimates for Outgoing Solutions of the Helmholtz Equation}

A resonance for the operator $H=-\Delta+V(x)$ on $L^{2}\left(\mathbb{R}^{3} \backslash \Omega\right)$ with supp $V \subset \mathscr{B}_{R}$ is a pole $k$ in the lower half-plane for Green's function, analytically continued in $k$ from the upper half-plane, or equivalently, a value of $k$ for which the integral equation

$$
\psi(x)=-\frac{1}{4 \pi} \int_{\mathscr{B}_{\rho} \backslash \Omega \mid} \frac{e^{i k|x-y|}}{|x-y|} V(y) \psi(y) d y-\frac{1}{4 \pi} \int_{\partial \Omega} \frac{e^{i k|x-y|}}{|x-y|} \frac{\partial \psi}{\partial v} d S
$$

has a solution. The expression $f(k, x)$ on the right-hand side of (3.1) makes sense for any $k \in \mathbb{C}$, and satisfies the Helmholtz equation $\Delta f+k^{2} f=0$ for $|x|>R$. It follows that $f(k, \cdot)$ is smooth in this region, so its expansion in spherical harmonics

$$
f(k, x)=\sum_{l=0}^{\infty} \sum_{|m| \leqq l}|x| \varphi_{l m}(k,|x|) Y_{l m}(\hat{x})
$$

is rapidly convergent. Here $\varphi_{l m}$ satisfies

$$
-\frac{\partial^{2} \varphi_{l m}(k, r)}{\partial r^{2}}+\frac{l(l+1)}{r^{2}} \varphi_{l m}(k, r)=k^{2} \varphi_{l m}(k, r)
$$

and is analytic in $k$ and $r$.

For $k$ in the upper half-plane $e^{i k|x-y|} /|x-y|$ decays exponentially as $|x| \rightarrow \infty$, so each $\varphi_{l m}(k, r)$ must be a decaying solution of (3.3); in fact

where

$$
\varphi_{l m}(k, r)=C w_{l}(k r)
$$

$$
w_{l}(z)=\sum_{j=0}^{l} \frac{(l+j) \cdots(l-j+1)}{j !(-2 i z)^{j}} e^{i z} .
$$

By analyticity, (3.4) and (3.5) hold for all $k$.

We seek to estimate $(\partial / \partial r-i k)(r \psi)$. Because of its exponential growth, $\psi$ is difficult to handle directly. Instead, we make a complex change of variables, which is the basic idea of complex scaling $[R-S]$. Consider

$$
h_{l}(s)=w_{l}\left(k\left(\rho+e^{i \theta} s\right)\right)
$$

where $k=|k| \exp (-i \theta)$. Then $h_{l}$ satisfies

$$
-h_{l}^{\prime \prime}(s)+\frac{l(l+1)}{\left(\rho e^{-i \theta}+s\right)^{2}} h_{l}(s)=|k|^{2} h_{l}(s), \quad(s>0)
$$


and

$$
h_{l}^{\prime}(s)-i|k| h_{l}(s) \rightarrow 0 \quad \text { as } \quad s \rightarrow \infty
$$

by (3.5). Thus we have traded a real potential and complex eigenvalue for a complex potential and positive eigenvalue. The following result on ordinary differential equations provides the necessary estimate on $\varphi_{l}$.

Proposition 3.1. Suppose that $K>0$ and $q$ is an integrable complex-valued continuously differentiable function on $[0, \infty)$ satisfying

$$
\begin{gathered}
\operatorname{Re} q^{\prime}+\frac{\operatorname{Im} q}{K}\left(\operatorname{Re} q+K^{2}\right) \leqq 0, \\
\operatorname{Im} q \geqq 0, \quad \operatorname{Re} q \geqq 0
\end{gathered}
$$

If $h$ satisfies

$$
\begin{gathered}
-h^{\prime \prime}+q h=K^{2} h, \\
h^{\prime}(s)-i K h(s) \rightarrow 0 \quad \text { as } \quad s \rightarrow \infty,
\end{gathered}
$$

then

$$
\begin{aligned}
A(s) & :=\left|h^{\prime}(s)-i K h(s)\right|^{2}-\operatorname{Re} q(s)|h(s)|^{2} \\
& =\left|h^{\prime}(s)\right|^{2}+\left(K^{2}-\operatorname{Re} q(s)\right)|h(s)|^{2}-2 K \operatorname{Im} h^{\prime}(s) \bar{h}(s) \leqq 0 .
\end{aligned}
$$

Proof. The equality in (3.12) is an algebraic identity. To prove the inequality we calculate

$$
\begin{aligned}
A^{\prime}= & h^{\prime \prime} \overline{h^{\prime}}+h^{\prime} \overline{h^{\prime \prime}}+\left(K^{2}-\operatorname{Re} q\right)\left(h^{\prime} \bar{h}+h \overline{h^{\prime}}\right)-\operatorname{Re} q^{\prime}|h|^{2}-2 K \frac{h^{\prime \prime} \bar{h}-h \overline{h^{\prime \prime}}}{2 i} \\
= & 2 \operatorname{Im} q \cdot \operatorname{Im} h^{\prime} \bar{h}-\operatorname{Re} q^{\prime}|h|^{2}-2 K \operatorname{Im} q|h|^{2} \\
= & \frac{\operatorname{Im} q}{K}\left[2 K \operatorname{Im} h^{\prime} \bar{h}-\left|h^{\prime}\right|^{2}-\left(K^{2}-\operatorname{Re} q\right)|h|^{2}\right] \\
& +\frac{\operatorname{Im} q}{K}\left|h^{\prime}\right|^{2}-\frac{\operatorname{Im} q}{K}\left(K^{2}+\operatorname{Re} q\right)|h|^{2}-\operatorname{Re} q^{\prime}|h|^{2} \\
\geqq & \frac{-\operatorname{Im} q}{K} A
\end{aligned}
$$

by (3.8) and (3.9). Thus

$$
A(s) \exp \int_{0}^{s} \frac{\operatorname{Im} q(t)}{K} d t
$$

increases with $s$, and by (3.11) and (3.9), it must approach a nonpositive value as $s \rightarrow \infty$. Therefore $A(s) \leqq 0$ for $s \leqq 0$.

Corollary 3.2. Let $k=\kappa-i \eta$. If

$$
\left(1+\frac{\eta^{2}}{\kappa}+\frac{l(l+1)}{\kappa^{2} \rho^{2}}\right) \eta \rho+3 \frac{\eta^{2}}{\kappa^{2}} \leqq 1,
$$


then

$$
\left|\varphi_{l m}^{\prime}(\rho)-i k \varphi_{l m}(\rho)\right|^{2} \leqq \frac{k^{2}}{|k|^{2} \rho^{2}} l(l+1)\left|\varphi_{l m}(\rho)\right|^{2} .
$$

Proof. First note that Proposition 3.1 applies to $h_{l}$ with $K=|k|$. For (3.11) holds by (3.7), and we have, writing $x=\rho \cos \theta=\rho \kappa /|k|$ and $y=\rho \sin \theta=\rho \eta /|k|$,

$$
q(s)=\frac{l(l+1)}{(x-i y+s)^{2}}=\frac{l(l+1)\left[(x+s)^{2}-y^{2}+2 i(x+s) y\right]}{\left((x+s)^{2}+y^{2}\right)^{2}},
$$

so that (3.9) holds, since (3.13) implies $x^{2}>y^{2}$, and

$$
\begin{aligned}
\operatorname{Re} q^{\prime} & +\kappa \operatorname{Im} q+\frac{\operatorname{Re} q \operatorname{Im} q}{\kappa} \\
= & \frac{l(l+1)}{\left((x+s)^{2}+y^{2}\right)^{2}}\left\{\left[2(x+s)-\frac{4(x+s)\left[(x+s)^{2}-y^{2}\right]}{(x+s)^{2}+y^{2}}\right]+2|k|(x+s) y\right. \\
& \left.+\frac{2(x+s) y}{|k|} \frac{l(l+1)\left((x+s)^{2}-y^{2}\right)}{\left((x+s)^{2}+y^{2}\right)^{2}}\right\} \\
= & \frac{2 l(l+1)(x+s)}{\left((x+s)^{2}+y^{2}\right)^{3}}\left\{3 y^{2}-(x+s)^{2}+|k| y\left((x+s)^{2}+y^{2}\right)+\frac{y}{|k|} l(l+1) \frac{(x+s)^{2}-y^{2}}{(x+s)^{2}+y^{2}}\right\} \\
\leqq & \frac{2 l(l+1)(x+s)}{\left((x+s)^{2}+y^{2}\right)^{3}}\left\{(3+|k| y) y^{2}-(1-|k| y)(x+s)^{2}+\frac{y}{|k|} l(l+1)\right\} .
\end{aligned}
$$

This does not exceed 0 if

$$
(1-|k| y) x^{2} \geqq(3+|k| y) y^{2}+\frac{y}{|k|} l(l+1),
$$

since $y=\rho \eta /|k|$ and $x=\rho \kappa /|k|$, this condition is

$$
(1-\eta \rho) \kappa^{2} \geqq(3+\eta \rho) \eta^{2}+\eta \rho \frac{l(l+1)}{\rho^{2}},
$$

which is equivalent to (3.13).

Therefore

$$
\begin{aligned}
0 & \geqq\left|h^{\prime}-i \kappa h\right|^{2}-\operatorname{Re} q|h|^{2} \\
& =|| k\left|w_{l}^{\prime}(k \rho)-i\right| k\left|w_{l}(k \rho)\right|^{2}-\frac{l(l+1)\left(k^{2} \rho^{2}-\eta^{2} \rho^{2}\right)}{|k|^{2} \rho^{4}}\left|w_{l}(k \rho)\right|^{2} \\
& \geqq C\left(\left|\varphi_{l m}^{\prime}(\rho)-i k \varphi_{l m}(\rho)\right|^{2}-\frac{l(l+1) \kappa^{2}}{|k|^{2} \rho^{2}}\left|\varphi_{l m}(\rho)\right|^{2}\right) .
\end{aligned}
$$

\section{Estimates Based on the Integral Equation}

The results of Sect. 3 do not hold for arbitrarily large $l$ and fixed $k$. In this case we must turn to the integral equation (3.1). If $\phi(x)=r \psi(x)$ and $\varphi$ is expanded in 
spherical harmonics $Y_{l m}$,

$$
\varphi(x)=\sum_{l=1}^{\infty} \sum_{|m| \leqq l} \varphi_{l m}(|x|) Y_{l m}(\hat{x})
$$

then (3.1) becomes

$$
\varphi_{l m}(r)=w_{l}(k r)\left\{\int_{\mathscr{B}_{R} \backslash \Omega} \frac{u_{l}(k|x|) Y_{l m}(\hat{x}) V(x) \psi(x) d x}{i^{l} k|x|}+\int_{i \Omega} \frac{u_{l}(k|x|) Y_{l m}(\hat{x}) \nabla \psi \cdot v d S}{i^{l} k|x|}\right\},
$$

where

$$
u_{l}(z)=\sum_{j=0}^{\infty} \frac{(-1)^{j} z^{l+2 j+1} 2^{l}(l+j) !}{(2 l+2 j+1) ! j !}=\frac{(2 z)^{l} z l !}{(2 l+1) !}-\cdots
$$

and

$$
w_{l}(z)=\sum_{j=0}^{l} \frac{(l+j) \cdots(l-j+1)}{j !(-2 i z)^{j}} e^{i z} .
$$

Proposition 4.1. Suppose that $\Omega \subset \mathscr{B}_{R_{0}}$ is star-shaped, and $R_{0} \leqq R<\rho$. If $\psi$ satisfies (3.1), and $\varphi=r \psi$ satisfies (4.1), then

$$
\begin{aligned}
& \sum_{|m| \leqq l}\left|\varphi_{l m}^{\prime}(\rho)-i k \varphi_{l m}(\rho)\right|^{2} \\
& \quad \leqq \frac{e^{4 \eta \rho}}{2}\left(1+\frac{e|k| \rho}{2 l}\right)^{2 l-2}\left[\left(\frac{R}{\rho}\right)^{2 l+2} R \int_{\mathscr{O S O}_{R} \backslash \Omega}|V \psi|^{2} d x+\left(\frac{R_{0}}{\rho}\right)^{2 l+2} \int_{\partial \Omega}\left|\frac{\partial \psi}{\partial v}\right|^{2} \frac{d S}{\hat{x} \cdot v}\right] .
\end{aligned}
$$

Proof. We need to estimate the kernel in (4.2). Since

$$
v_{l}(z):=u_{l}(z) / z^{l+1}
$$

satisfies

$$
v^{\prime \prime}+\frac{l+1}{z} v^{\prime}+v=0
$$

we have

$$
\frac{d^{2} v}{d|z|^{2}}+\frac{l+1}{|z|} \frac{d v}{d|z|}+e^{2 i \arg z} v=0
$$

so

$$
\begin{aligned}
\frac{d}{d|z|}\left(\left|\frac{d v}{d|z|}\right|^{2}+|v|^{2}\right) & =-2 \frac{l+1}{|z|}\left|\frac{d v}{d|z|}\right|^{2}+2 \operatorname{Re}\left(\left(1-e^{2 \arg z}\right) v \frac{d v}{d|z|}\right) \\
& \leqq\left|1-e^{2 \arg z}\right|\left(\left|\frac{d v}{d|z|}\right|^{2}+|v|^{2}\right) \\
& \leqq 2\left|\frac{\operatorname{Im} z}{z}\right|\left(\left|\frac{d v}{d|z|}\right|^{2}+|v|^{2}\right) .
\end{aligned}
$$


Since

$$
v_{l}(0)=2^{l} l ! /(2 l+1) ! \quad \text { and } \quad v_{l}^{\prime}(z)=O\left(z^{2}\right),
$$

it follows that $\left|v_{l}(z)\right|^{2} \leqq \exp (2|\operatorname{Im} z|)\left(2^{l} l ! /(2 l+1) !\right)^{2}$ and

$$
\left|u_{l}(z)\right| \leqq\left(|z||2 z|^{l} l ! /(2 l+1) !\right) \exp |\operatorname{Im} z| .
$$

Therefore, if $0<\alpha<1$,

$$
e^{2|\operatorname{Im} z|}\left|e^{-i z}\left(w_{l}^{\prime}-i \omega_{l}(z)\right) u_{l}(\alpha z)\right| \leqq \frac{|z| \alpha^{l+1}}{2 l+1} \sum_{j=0}^{l}\left(\begin{array}{l}
l \\
j
\end{array}\right) \frac{j}{|z|} \frac{|2 z|}{2 l} \cdots \frac{|2 z|}{l+j+1} .
$$

Now

$$
\begin{aligned}
\frac{1}{l-j} \log \left(\frac{1}{2 l} \cdots \frac{1}{l+j+1}\right) & \leqq-\frac{1}{l-j_{l+j}} \log x d x \\
& \leqq-\frac{1}{l} \int_{l}^{2 l} \log x d x=1-2 \log 2-\log l
\end{aligned}
$$

so

$$
\frac{|2 z|}{2 l} \cdots \frac{|2 z|}{l+j+1} \leqq\left(\frac{e|z|}{2 l}\right)^{l-j}
$$

Thus we have

$$
\begin{aligned}
& e^{2|\operatorname{Im} z|}\left|e^{-i z}\left(w_{l}^{\prime}(z)-i \omega_{l}(z)\right) u_{l}(\alpha z)\right| \\
& \quad \leqq-\frac{\alpha^{l+1}|z|}{2 l+1}\left(\frac{e|z|}{2 l}\right)^{l} \frac{d}{d|z|}\left(1+\frac{2 l}{e|z|}\right)^{l} \\
& =\frac{\alpha^{l+1} l}{2 l+1}\left(1+\frac{e|z|}{2 l}\right)^{l-1} \leqq \frac{\alpha^{l+1}}{2}\left(1+\frac{e|z|}{2 l}\right)^{l-1} .
\end{aligned}
$$

Using the fact that, by Bessel's inequality,

$$
\sum_{|m| \leqq l}\left|\int_{S^{2}} Y_{l m}(\hat{x}) f(\hat{x}) d S(\hat{x})\right|^{2} \leqq \int_{S^{2}}|f(\hat{x})|^{2} d S(\hat{x})
$$

we have, taking $\alpha=R / \rho$ and $R_{0} / \rho$

$$
\begin{aligned}
& \sum_{|m| \leqq l}\left|\varphi^{\prime}(\rho)-i k \varphi(\rho)\right|^{2} \\
& =\left|w_{l}^{\prime}(k \rho)-i \omega_{l}(k \rho)\right|^{2} \sum_{|m| \leqq l} \mid \int_{\mathscr{B}_{R} \backslash \Omega} \frac{u_{l}(k|x|) Y_{l m}(\hat{x}) V(x) \psi(x)}{|x|} d x \\
& \quad+\left.\int_{\partial \Omega} \frac{u_{l}(k|x|) Y_{l m}(\hat{x}) \partial \psi(x) / \partial v}{|x|} d S\right|^{2}
\end{aligned}
$$




$$
\begin{aligned}
\leqq & \frac{e^{4 \eta \rho}}{2}\left(1+\frac{e|k| \rho}{2 l}\right)^{2 l-2} \sum_{|m| \leqq l}\left\{\left(\frac{R}{\rho}\right)^{2 l+2}\left|\int_{\mathscr{B}_{R} \backslash \Omega} Y_{l m}(\hat{x}) V(x) \psi(x) d x\right|^{2}\right. \\
& \left.+\left(\frac{R_{0}}{\rho}\right)^{2 l+2}\left|\int_{S^{2}} Y_{l m}(\hat{x}) \frac{\partial \psi}{\partial v} \frac{d S(\hat{x})}{\hat{x} \cdot v}\right|^{2}\right\} \\
\leqq & \frac{e^{4 \eta \rho}}{2}\left(1+\frac{e|k| \rho}{2 l}\right)^{2 l-2}\left\{\left(\frac{R}{\rho}\right)^{2 l+2} R \int_{\mathscr{B} R \backslash \Omega}|V(x) \psi(x)|^{2} d x\right. \\
& \left.+\left(\frac{R_{0}}{\rho}\right)^{2 l+2} \int_{\partial \Omega}\left|\frac{\partial \psi}{\partial v}\right|^{2} \frac{d S}{\hat{x} \cdot v}\right\} .
\end{aligned}
$$

Proposition 4.2. Suppose that $\varphi_{l m}$ is as in Proposition 4.1, let $\delta>0$ and

$$
\beta=1+\frac{e}{2}\left[\delta\left(1+\frac{\sqrt{2 \delta}}{\kappa R}\right)\right]^{1 / 2}
$$

and suppose $\beta R / \rho<1$. Denote by $l_{0}$ the smallest positive integer such that $l_{0}\left(l_{0}+1\right) \delta \geqq|k|^{2} \rho^{2}$. Set

$$
I=R \int_{\mathscr{B}_{\boldsymbol{R}} \backslash \Omega}|V \psi|^{2} d x, \quad I_{0}=\int_{\partial \Omega}\left|\frac{\partial \psi}{\partial v}\right|^{2} \frac{d S}{\hat{x} \cdot v} .
$$

Then

$$
\sum_{l=l_{0}}^{\infty} \sum_{|m| \leqq l}\left|\varphi_{l m}^{\prime}(\rho)-i k \varphi_{l m}(\rho)\right|^{2} \leqq \frac{e^{2 \eta \rho}}{2 \beta^{4}}\left[\frac{I\left(\beta \frac{R}{\rho}\right)^{2+\sqrt{2 / \delta}|\kappa| \rho}}{1-\left(\beta \frac{R}{\rho}\right)^{2}}+\frac{I_{0}\left(\beta \frac{R_{0}}{\rho}\right)^{2+\sqrt{2 / \delta}|\kappa| \rho}}{1-\left(\beta \frac{R_{0}}{\rho}\right)^{2}}\right] .
$$

Proof. By (4.5) the quantity on the left-hand side of (4.6) does not exceed

$$
\begin{aligned}
& \sum_{l=l_{0}}^{\infty} \frac{1}{2} e^{4 \eta \rho}\left(1+\frac{e|k| \rho}{2 l}\right)^{2 l-2}\left[I\left(\frac{R}{\rho}\right)^{2 l+2}+I_{0}\left(\frac{R_{0}}{\rho}\right)^{2 l+2}\right] \\
& \leqq \frac{1}{2} \frac{e^{4 \eta \rho}}{\beta^{4}} \sum_{l=l_{0}}^{\infty}\left[I\left(\frac{\beta R}{\rho}\right)^{2 l+2}+I_{0}\left(\frac{\beta R_{0}}{\rho}\right)^{2 l+2}\right] \\
& \quad \leqq \frac{1}{2} \frac{e^{4 \eta \rho}}{\beta^{4}}\left[\frac{I\left(\frac{\beta R}{\rho}\right)^{2 l_{0}+2}}{1-\left(\frac{\beta R}{\rho}\right)^{2}}+\frac{\left.I_{0}\left(\frac{\beta R_{0}}{\rho}\right)^{2 l_{0}+2}\right]}{1-\left(\frac{\beta R_{0}}{\rho}\right)^{2}}\right] .
\end{aligned}
$$

We have used $|k| \rho / l \leqq \sqrt{\delta(1+(1 / l))} \leqq \sqrt{2 \delta}$ for $l \geqq l_{0}$, which implies

$$
\begin{aligned}
2 l_{0}+2 & \geqq 2+(2 / \delta)^{1 / 2}|k| \rho \\
& \geqq 2+(2 / \delta)^{1 / 2} \kappa \rho
\end{aligned}
$$


and

$$
\begin{aligned}
1+\frac{e|k| \rho}{2 l} & \leqq 1+\frac{e}{2}\left[\delta\left(1+\frac{1}{l}\right)\right]^{1 / 2} \\
& \leqq 1+\frac{e}{2}\left\{\delta\left(1+\frac{(2 \delta)^{1 / 2}}{|k| \rho}\right)\right\}^{1 / 2} \\
& \leqq 1+\frac{e}{2}\left\{\delta\left(1+\frac{(2 \delta)^{1 / 2}}{\kappa R}\right)\right\}^{1 / 2}=\beta
\end{aligned}
$$

\section{Lower Bounds for Resonance Widths}

Now the results of Sects. 2,3,4 can be combined to bound $\eta=-\operatorname{Im} k$ below. The first theorem deals with a Schrödinger operator at energies where classical trapping does not happen. The second obtains (exponentially small) lower bounds in trapping situations where we do expect resonances near the real axis. The third treats the Laplacian in the exterior of a star-shaped obstacle.

Theorem 5.1. Suppose that $V \in \mathscr{C}^{1}\left(\mathbb{R}^{3}\right)$ with $V(x)=0$ for $|x| \geqq R$. The Schrödinger operator $H=-\Delta+V$ on $L^{2}\left(\mathbb{R}^{3}\right)$ has no resonance at $k=\kappa-i \eta, \kappa, \eta>0$ if

$$
v=\inf \left\{\kappa^{2}-V(x)-r \frac{\partial V(x)}{\partial r}:|x| \leqq R\right\}>0,
$$

and for some $\alpha \in(0,1)$

$$
\eta R<\frac{\alpha \frac{v}{\kappa^{2}}\left[1-\frac{\alpha}{1-\alpha^{2}} \alpha^{\sqrt{2 \beta} \beta R} b\right]-3\left(\frac{\alpha}{2 \beta \kappa R}\right)^{2}}{2 A \beta}
$$

where

$$
\begin{aligned}
& \beta=1+\frac{e}{2}\left[1+\frac{2}{\kappa R}\right]^{1 / 2}, \\
& b=\frac{e^{2} R^{2}}{2 \beta^{3}} \sup \left\{\frac{V(x)^{2}}{\kappa^{2}-V(x)-\left(r \frac{\partial V}{\partial r}\right)}:|x| \leqq R\right\} \\
& A=\sup \left\{1+\frac{V(x)}{\kappa^{2}}+\left(\frac{\alpha}{2 \beta \kappa R}\right)^{2}:|x| \leqq R\right\}
\end{aligned}
$$

Remark. As $\kappa \rightarrow \infty, v / \kappa^{2} \rightarrow 1, \beta \rightarrow 1+e / 2, b \rightarrow 0$, and $A \rightarrow 1$, so for any $\alpha<1$ the limiting width of the resonance-free region given by $(5.2)$ is

$$
\eta R<\frac{\alpha}{2}(1+e / 2)^{-1} \sim(4.718)^{-1} \alpha .
$$

A resonance for $-\hbar^{2} \Delta+V$ at $k$ is equivalent to one for $-\Delta+V \mid \hbar^{2}$ at $k / \hbar$. As 
$\hbar \rightarrow 0$, we get asymptotically for the resonance-free region

$$
\eta R<\frac{\alpha \hbar v / \kappa^{2}}{2\left\|1+V / \kappa^{2}\right\|_{\infty}(1+e / 2)} .
$$

Proof of Theorem 5.1. Suppose that $\psi$ is a resonance eigenfunction and the resonance $k$ satisfies (5.1) and (5.2). Then $\varphi=r \psi$ satisfies (2.12) with $\Omega=\varnothing$. In (2.12) set $\rho=\beta R / \alpha$. Then by (5.2), $2 \eta \rho=2 \eta \beta R / \alpha<1$, and we have

$$
\begin{gathered}
\int_{\mathscr{B}_{\rho}}\left[\kappa^{2}(1-2 \eta \rho)-\left(V+\eta^{2}\right)(1+2 \eta \rho)-r \frac{\partial V}{\partial r}\right] \frac{|\varphi|^{2}}{r^{2}} d x \\
\leqq \frac{1}{\rho_{r}} \int_{r=\rho}\left\{\left|\frac{\partial \varphi}{\partial r}-i k \varphi\right|^{2}-\left(|\nabla \varphi|^{2}-\left|\frac{\partial \varphi}{\partial r}\right|^{2}\right)\right\} d S \\
=\rho \sum_{l=0}^{\infty} \sum_{|m| \leqq l}\left\{\left|\varphi_{l m}^{\prime}-i k \varphi_{l m}\right|^{2}-\frac{l(l+1)}{\rho^{2}}\left|\varphi_{l m}\right|^{2}\right\},
\end{gathered}
$$

where $\varphi_{l m}$ are defined by (3.2). By Corollary 3.2, the terms with $l(l+1) \leqq|k|^{2} \rho^{2}=$ $\left(\kappa^{2}+\eta^{2}\right) \rho^{2}$ in this series are all less than or equal to zero if

$$
\left(1+\frac{\eta^{2}}{\kappa^{2}}\right) \frac{2 \eta \beta R}{\alpha}+3 \frac{\eta^{2}}{\kappa^{2}} \leqq 1 .
$$

The remaining terms in the series in (5.4) may be estimated by Proposition 4.2, taking $\delta=1$, since $\beta R / \rho=\alpha<1$, giving

$$
\begin{aligned}
\int_{\mathscr{B}_{\rho}} & {\left[\kappa^{2}(1-2 \eta \rho)-\left(V+\eta^{2}\right)(1+2 \eta \rho)-r \frac{\partial V}{\partial r}\right] \frac{|\varphi|^{2}}{r^{2}} d x } \\
& \leqq \frac{e^{4 \eta \rho}}{2 \beta^{3}} \frac{(\beta R / \rho)^{1+\sqrt{2}|\kappa| \rho}}{1-(\beta R / \rho)^{2}} R^{2} \int_{\mathscr{B}_{R}} \frac{|V \varphi|^{2}}{r^{2}} d x .
\end{aligned}
$$

But this inequality is impossible, and (5.5) holds if for $|x|<\rho$,

$$
\begin{aligned}
& 3\left(\frac{\alpha}{2 \beta \kappa R}\right)^{2}+\left(1+\frac{V(x)}{\kappa^{2}}+\left(\frac{\alpha}{2 \beta \kappa R}\right)^{2}\right) \frac{2 \beta \eta R}{\alpha}+\frac{e^{2}(R V(x))^{2} \alpha^{\sqrt{2} \kappa R}}{2 \beta^{3} \kappa^{2}} \frac{\alpha}{1-\alpha^{2}} \\
& \quad \leqq \frac{\kappa^{2}-V(x)-r \frac{\partial V}{\partial r}(x)}{\kappa^{2}} .
\end{aligned}
$$

We have used $\exp (4 \eta \rho) \leqq e^{2}$ and

$$
\frac{\eta}{\kappa} \leqq \frac{\alpha}{2 \beta \kappa R},
$$

which follows from $2 \eta \rho<1$. But (5.7) follows from (5.2), giving a contradiction.

Theorem 5.2. Suppose that $V \in \mathscr{C}^{1}\left(\mathbb{R}^{3}\right)$ and $\operatorname{supp} V \subset \mathscr{B}_{R}$, and let $R_{1}, q(r)$ and $B$ be defined as in Theorem 2.6. The Schrödinger operator $H=-\Delta+V$ on $L^{2}\left(\mathbb{R}^{3}\right)$ has 
no resonance at $k=\kappa-$ in if $\kappa R>1$ and

$$
8 \eta R_{2} B \leqq 1
$$

where

$$
R_{2}=\max \left\{\|V\|_{\infty} R / 2 \kappa^{2}, R_{1}, e \beta R\right\}
$$

and $\beta$ is defined by (5.3).

Proof. Suppose that $k$ is a resonance satisfying (5.9) and let $\psi$ be the corresponding resonance eigenfunction. Then $\varphi=r \psi$ satisfies (2.16) with $\rho=R_{2}$, and since $4 \eta R_{2} B<1$ we have

$$
\begin{aligned}
& \int_{\mathscr{B}_{R_{2}}}\left(\frac{\kappa^{2}}{2}-\eta^{2}-2 \eta R_{2} \kappa^{2} B\right)|\varphi|^{2} \frac{d x}{r^{2}} \\
& \quad \leqq B R_{2} \sum_{l=0}^{\infty} \sum_{|m| \leqq l}\left\{\left|\varphi_{l m}^{\prime}\left(R_{2}\right)-i k \varphi_{l m}\left(R_{2}\right)\right|^{2}-\frac{l(l+1)}{R_{2}^{2}}\left|\varphi_{l m}\left(R_{2}\right)^{2}\right|\right\} .
\end{aligned}
$$

The terms in this series with $l(l+1) \leqq B|k|^{2} R_{2}^{2}$ are negative by Corollary 3.2 since (3.13) holds:

$$
\begin{aligned}
\left(1+\frac{\eta^{2}}{\kappa^{2}}\right)(1+B) \eta R_{2}+\frac{3 \eta^{2}}{\kappa^{2}} & \leqq\left(1+\frac{1}{\left(8 \kappa R_{2} B\right)^{2}}\right)(1+B) \eta R_{2}+\frac{3}{\left(8 \kappa R_{2} B\right)^{2}} \\
& \leqq\left(1+\frac{1}{64}\right)(1+B) \eta R_{2}+\frac{3}{64} \leqq 1,
\end{aligned}
$$

by (5.9).

Because $R_{2} \geqq e \beta R>\beta R$, the remaining terms in the series can be estimated by Proposition 4.2 with $\delta=B^{-1}$, giving

$$
\int_{\mathscr{B}_{R_{2}}}\left(\frac{\kappa^{2}}{2}-\eta^{2}-2 \eta R_{2} B \kappa^{2}\right)|\varphi|^{2} \frac{d x}{r^{2}} \leqq \frac{B e^{1 / 2}}{2 \beta^{3}} \frac{\left(\beta R / R_{2}\right)^{1+\sqrt{2} B|k| R_{2}}}{1-\left(\beta R / R_{2}\right)^{2}} R^{2} \int_{\mathscr{B}_{R}}|V \varphi|^{2} \frac{d x}{r^{2}}
$$

Now since the maximum value of the function $x^{2} e^{-x}$ is $4 / e^{2}$ so that $e^{-x} \leqq 4 /(e x)^{2}$, we have

$$
\begin{aligned}
B\left(\beta R / R_{2}\right)^{(2 B)^{1 / 2}|k| R_{2}} & \leqq \frac{2}{\left|e k r_{2}\right|^{2} \log ^{2}\left(R_{2} / \beta R\right)} \\
& \leqq \frac{8 \kappa^{2}}{e^{2}\|V\|_{\infty}^{2} R^{2}}
\end{aligned}
$$

by the definition of $R_{2}$. Since $\beta R / R_{2}<1 / e$, we have

$$
\frac{\beta R / R_{2}}{1-\left(\beta R / R_{2}\right)^{2}} \leqq \frac{1}{e\left(1-e^{-2}\right)} \leqq \frac{1}{2}
$$

These estimates with (5.11) give

$$
\frac{\kappa^{2}}{2}-\eta^{2}-2 \eta R_{2} B \kappa^{2} \leqq \frac{2 \kappa^{2}}{e^{3 / 2} \beta^{3}}
$$


But (5.9) implies

$$
2 \eta R_{2} B+\frac{\eta^{2}}{\kappa^{2}}+\frac{2}{e^{3 / 2} \beta^{3}} \leqq \frac{1}{4}+\frac{1}{(8 \kappa R)^{2}}+\frac{2}{e^{3 / 2} \beta^{3}}<\frac{1}{2}
$$

which contradicts (5.12).

Theorem 5.3. Let $\Omega \subset \mathscr{B}_{R} \subset \mathbb{R}^{3}$ be a domain with $\mathscr{C}^{1}$ boundary, which is star-shaped. Then the Laplacian with Dirichlet boundary condition on $\partial \Omega$ has no resonance at $k=\kappa-i \eta, \kappa, \eta>0$ if

where $\alpha$ is defined by

$$
\eta R<\frac{\alpha}{2 \beta} \frac{1-3 \frac{1}{(2 \beta \kappa R)^{2}}}{1+\frac{1}{(2 \beta \kappa R)^{2}}}
$$

$$
\frac{\alpha^{1+\sqrt{2} \kappa \beta R}}{1-\alpha^{2}}=\frac{2 \beta^{3} \inf \left\{(x \cdot v)^{2}: x \in \partial \Omega\right\}}{(e R)^{2}}
$$

and $\beta$ is defined by (5.3).

Remark. As $\kappa \rightarrow \infty$, it follows from (5.14) that $\alpha \rightarrow 1$, so the lower bound on $\eta R$ given by $(5.13)$ approaches $(2+e)^{-1}$.

Proof. If $\psi$ is a resonance eigenfunction with resonance $k$ satisfying (5.13) then $\varphi=r \psi$ satisfies (2.12), and if $\rho>R \beta$ and

$$
\left(1+\frac{\eta^{2}}{\kappa^{2}}\right) 2 \eta \rho+3 \frac{\eta^{2}}{\kappa^{2}} \leqq 1
$$

the integrals over $\mathscr{B}_{\rho} \backslash \Omega$ in (2.12) are nonnegative and we obtain, using Corollary 3.2 and Proposition 4.2,

$$
\begin{aligned}
\int_{\partial \Omega} x \cdot v|\nabla \varphi|^{2} \frac{d S}{r^{2}} & <\frac{e^{2 \eta \rho}}{2 \beta^{3}} \frac{(\beta R / \rho)^{\sqrt{2}} \kappa \rho+1}{1-(\beta R / \rho)^{2}} R \int_{\partial \Omega} \frac{|\nabla \varphi|^{2} d S}{r x \cdot v} \\
& \leqq \frac{e^{2}}{2 \beta^{3}} \frac{(\beta R / \rho)^{\sqrt{2} \kappa R+1}}{1-(\beta R / \rho)^{2}} \frac{R^{2}}{\inf (x \cdot v)^{2}} \int_{\partial \Omega}|\nabla \varphi|^{2} x \cdot v \frac{d S}{r^{2}}
\end{aligned}
$$

The function on the left side of (5.14) increases from 0 to $\infty$ as $\alpha$ runs from 0 to 1 , so $(5.14)$ has a unique solution in $(0,1)$, and taking $\rho=\beta R / \alpha$ makes $(5.16)$ impossible, and (5.15) follows from (5.13), which gives a contradiction.

Acknowledgements. We are grateful to Walter Strauss and Peter Hislop for references to related work.

\section{References}

[B-L-R] Bardos, C., Lebeau, G., Rauch, J.: Scattering frequenceis and Gevrey 3 singularities. Invent. Math. 90, 77-114 (1987) 
[B-C-D] Briet, Ph., Combes, J.M., Duclos, P.: On the location of resonances for Schrödinger operators in the semiclassical limit I. Resonance free domains. J. Math. Anal. App. 126, 90-99 (1987)

[D-H] DeBièvre, S., Hislop, P.: Spectral resonances for the Laplace-Beltrami operator. Ann. Inst. H. Poincaré 48, 105-145 (1988)

[H] Harrell, E. M.: General lower bounds for resonances in one dimension. Commun. Math. Phys. 86, 221-225 (1982)

[LA-1] Lavine, R.: Commutators and scattering II; A class of one body problems, Ind. Univ. Math. J. 21, 643-656 (1972)

[LA-2] Lavine, R.: Spectral density and sojourn times. In: Nuttall, J. (ed.) Atomic scattering theory. London, Ontario: Univ. Western Ontario 1978, pp. 45-61

[LA-3] Lavine, R.: Constructive estimates in quantum scattering, unpublished

[LO] Loe, B.: Scattering by potentials of unbounded support, Ph.D. thesis, Brown University, to appear 1989

[M] Morawetz, C.: On the modes of decay for the wave equation in the exterior of a reflecting body. Proc. Roy. Irish Acad. C72, 113-120 (1972)

[M-R-S] Morawetz, C., Ralston, J. and Strauss, W.: Decay of solutions of the wave equation outside nontrapping obstacles. Commun. Pure Appl. Math. 30, 447-508 (1977)

[R] Ralston, J.: The first variation of the scattering matrix: An addendum. J. Diff. Equations 28, $155-162$ (1978)

[R-S] Reed, M., Simon, B.: Methods of modern mathematical physics IV: analysis of operators. New York: Academic Press 1978

[S] Sigal, I. M.: Sharp exponential bounds on resonance states and width of resonances. Adv. Appl. Math. 9, 127-166 (1988)

Communicated by B. Simon

Received June 23, 1989 